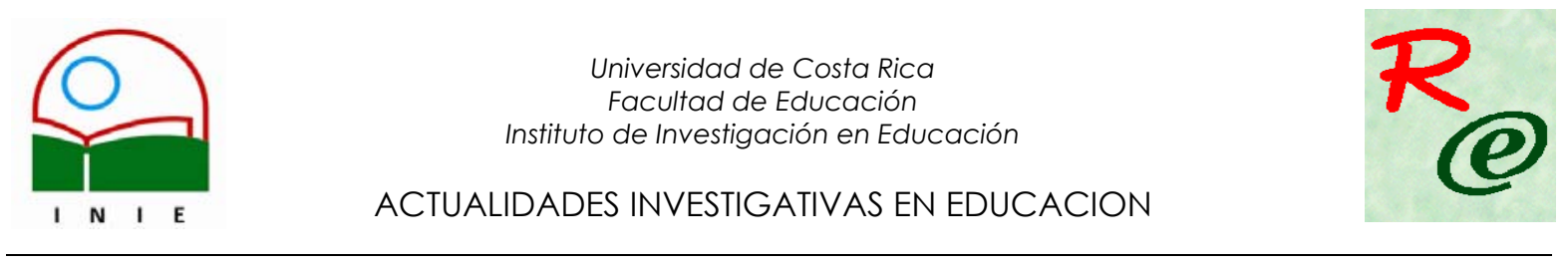

\title{
CONCEPCIONES ACERCA DE LA MATERNIDAD EN LA EDUCACIÓN FORMAL Y NO FORMAL
}

\begin{abstract}
Kathia Alvarado Calderón ${ }^{1}$
Resumen: Este artículo presenta algunos resultados de la investigación desarrollada en el Instituto de Investigación en Educación (INIE), bajo el nombre "Construcción del concepto de maternidad en la educación formal y no formal".

Utilizando un enfoque cualitativo de investigación, recurrimos a las técnicas de elaboración de dibujos, entrevistas y grupo focal como recursos para la recolección de la información. De esta manera, podemos acercarnos a las concepciones de la maternidad que utilizan los participantes de las diferentes instancias educativas (formal y no formal) con quienes se trabajó.

Palabras clave: EDUCACIÓN FORMAL/ EDUCACIÓN NO FORMAL/MATERNIDAD/ GÉNERO/

Abstract: This article presents some results the research developed in the Instituto de Investigación en Educación (INIE), named "Construcción del concepto de maternidad en la educación formal y no formal".

It begins with a theoretical analysis about social conceptions regarding motherhood in the occidental societies. Among the techniques for gathering information were thematic drawing, interview and focus group, using a qualitative approach research method. This is followed by a brief summary of main findings. The article concludes with a proposal of future working lines for the deconstruction of the motherhood concept in formal and informal education contexts.
\end{abstract}

Key words: FORMAL EDUCATION/ NON-FORMAL EDUCATION/MOTHERHOOD/ GENDER/

\section{Presentación}

Este artículo presenta la investigación desarrollada en el marco del Programa de Educación y Género del Instituto de Investigación en Educación (INIE), titulada "Construcción del concepto de maternidad en la educación formal y no formal". Inscrita como proyecto de investigación \# 724-A2-032, concluido en diciembre del 2004. A cargo de las investigadoras M.Sc. Ana Lucía Villarreal Montoya y la Licda. Kathia Alvarado Calderón.

\footnotetext{
${ }^{1}$ Licenciada en Psicología. Investigadora del Instituto de Investigación en Educación (INIE). Profesora de la Escuela de Orientación y Educación Especial; labora también en la Oficina de Orientación en el Centro de Asesoría Estudiantil (CASE) de la Facultad de Ciencias Sociales, ambos de la Universidad de Costa Rica.
}

Correo electrónico: kathiaa@cariari.ucr.ac.cr

Artículo recibido: 10 de mayo, 2005

Aprobado: 20 de junio, 2005 
Nuestra investigación tuvo como objetivo, develar la construcción social, política, ideológica y económica de la maternidad, desde la perspectiva de género. Pero también somos conscientes de la importancia de la escuela en la socialización de estos conceptos, y nos interesó trabajar con población infantil -niños y niñas de nueve años (9) correspondientes al tercer grado de la educación primaria-. Por tanto, analizar el discurso de la docente de estos niños y niñas, se consideró fundamental para nuestro trabajo. Por otra parte, quisimos abarcar el espacio de la educación no formal, y para ello, definimos como población meta, un grupo de mujeres ubicadas en el programa "Creciendo Juntas", del Instituto Mixto de Ayuda Social (IMAS).

Como objetivos generales, se plantearon los siguientes:

- Analizar el proceso de construcción del concepto de maternidad en la educación formal y no formal.

- Elaborar estrategias para deconstruir el concepto de maternidad.

\section{Referencia teórica.}

\subsection{Mitos sociales acerca de la maternidad: La maternidad como construcción social.}

Cuando nos referimos a mitos sociales, abarcamos con ello, las construcciones imaginarias que las sociedades necesitan para justificarse en su funcionamiento; pero estas significaciones no corresponden a elementos "racionales" o "reales", sino que están referidas a un acto de creación social. La sociedad organiza, de este modo, su propia forma de ver el mundo.

Cuando afirmamos que la maternidad es construida socialmente con referencia a mitos o imaginarios sociales, decimos que "maternidad" y "mujer" como conceptos han pasado por el mismo camino de atribución de significación.

Es central en esta construcción, la naturaleza y la función de la mujer, las cuales se encuentran asimiladas en la concepción sobre la maternidad. Desde Aristóteles en su trabajo Enciclopedia, se puede observar que el campo de la biología asigna a las mujeres y a los varones funciones generativas que se instalaron luego en el discurso social. Así, al hombre se le sitúa en el dominio del pensamiento y de la inteligencia (la causa formal, la final y la eficiente); mientras que para la mujer, el dominio se mantuvo del lado de la materia y la 
sustancia, como recipiente del acto de generación -procreación- (Vigetti, 1994, Olivier, C. 1994).

Estos imaginarios reforzados a lo largo de la historia de la cultura occidental: Grecia, Roma y el judeo-cristianismo, respaldan la concepción del aporte del hombre a la cultura, en tanto que la mujer continúa siendo ubicada del lado de lo doméstico, separada de la creación cultural entendida como creación simbólica.

De tal manera que la mujer es reducida al terreno de la "carne", excluida de lo intelectual, lo moral y lo filosófico. Esta visión la hereda el cristianismo, conservándola en la idea de la mujer inferior y carnal, consecuencia también del dualismo en el que se pensó el bien y el mal en el ser humano en San Agustín. En el matrimonio cristiano, cuyo fin estaba referido a la procreación de los hijos (as), deviene la salvación para quienes no pueden contener su deseo. Paradójicamente, es con el establecimiento del matrimonio religioso (S XI) y con la veneración de la Virgen María, en tanto Madre (S XII), cuando las mujeres alcanzan tímidamente un lugar en esta familia como producto social (Rodríguez, 1994).

En la Edad Media con las creaciones del amor cortés y la nueva condición que la mujer obtiene con el apelativo de Dama (mujer del señor feudal), ésta aparece en el panorama de la discusión acerca del matrimonio y del amor. Así nacen las expresiones líricas realizadas por las mujeres concernientes a este tema. La Dama pondría sus condiciones para convertirse en esposa y madre; el varón se esforzaría por cumplir y ser aceptado. Sin embargo, la mujer queda atrapada en el ideal del Eterno Femenino.

Con el Renacimiento, algunas fórmulas cambian, surge con Rousseau una nueva concepción del niño (a) y de sus necesidades afectivas desde el nacimiento y nuevos "contratos" se crean. Que el niño requiera de ser acariciado y tratado dulcemente, permite que se le asigne a la madre el papel de cuidar de él, mientras que el padre cuidaba su formación, su saber y su espíritu. Así vemos un "contrato de amor" para la mujer y un "contrato social" para el padre.

Con la Revolución Industrial se presenta una reorganización familiar, el padre debe salir de la casa para trabajar por jornadas muy largas y, cuando regresa agotado por su trabajo, maltrata a su esposa y a sus hijos (as). Para C. Olivier (1994), el padre es ubicado casi fuera 
del lazo familiar, pero al mismo tiempo, permanece en una especie de mito sostenido a través de la palabra de la mujer. Vemos en esta nueva organización de la familia, la responsabilidad de la crianza de los hijos (as) y de la familia en las manos maternas, hasta que los hijos (as) llegaran a la llamada edad de la razón (los 7 años).

La mujer es definida a partir de su función materna y su función de esposa, es decir, desde fuera de sí misma. Esto someterá a las mujeres en un proceso sistemático de desconocimiento de sí. Los sobreentendidos al respecto del proyecto de vida de la mujer, se dirigen como única salida a la realización de la maternidad, produciéndose el mito que organizará las relaciones en torno de la mujer en tanto que madre.

De esta forma, la mujer se encuentra privada, de su contribución activa en la generación humana, pero adquiere un reconocimiento social, en tanto ocupe el imaginario que se le asigna: cuidadora de hijos (as), del marido y de los demás. Asimismo, sufre una restricción de su desempeño al ámbito amoroso y sentimental como un asunto preferentemente femenino. En otras palabras, los conceptos mujer, madre, hija son en virtud de las significaciones imaginarias -arbitrarias- que la sociedad como colectivo anónimo, asigna a estos conceptos, estableciendo a un tiempo mitos para que quienes los encarnan cumplan con el encargo.

El mito sobre la maternidad y el Eterno Femenino se entienden como parte del sistema de interpretación que la sociedad ha elegido para ello. Ciertamente, las circunstancias a las que se enfrenta la mujer, producen contradicciones en la vivencia de la maternidad; la mujer se erige sobre una función idealizada como madre, pero entre incontables barreras para sentirse satisfecha en ella o en otras formas de realización femenina.

Es como si el "paradigma aristotélico" siguiera dominando las vidas de los hombres y de las mujeres, al tener excluida del pensamiento científico, la capacidad generativa femenina, no en términos de procreación, sino como creación que trasciende el acto de engendrar. No hay que olvidar que con Aristóteles, este desempeño de la mujer en la maternidad se circunscribe a una función que la involucra en cuanto materia, dejándole solo al hombre el poder generador. 
Como señalaron en su momento, De Simone y Saavedra (1992) la importancia de la reproducción ha sido un objetivo de vida impuesto para la mujer, lo que no le permite visualizarse como un "sujeto de deseo", con un proyecto de vida que no incluya necesariamente el ser madre, como consecuencia, su sexualidad y su feminidad quedan condicionadas al acontecimiento de la maternidad.

\subsection{La maternidad como construcción del patriarcado?}

\subsubsection{El cuerpo femenino}

Nuestra investigación se inscribe dentro de la perspectiva de género. Esto quiere decir que partimos de la premisa de que la sociedad actual es patriarcal y que, por lo tanto, las diversas instituciones y la sociedad, en general, se desempeñan como tales, reproduciendo el patriarcado como paradigma o forma de pensar y actuar, individual y colectivamente. La teoría feminista plantea la visibilización de la opresión de las mujeres en la sociedad patriarcal y, específicamente, en la vida privada.

A lo largo de ese proceso histórico patriarcal, se ha expropiado a las mujeres de su derecho de propiedad sobre la maternidad y sobre la sexualidad en general. Como señalamos en el apartado precedente, el patriarcado ejerce el poder sobre las mujeres, al reducirlas y limitarlas a cumplir el rol reproductivo.

Para Foucault (1998), el sexo es, a un tiempo, acceso a la vida del cuerpo y a la vida de la especie. Es utilizado como matriz de las disciplinas y principio de las regulaciones. Por ello, en el siglo XIX, la sexualidad era perseguida hasta en el más ínfimo detalle de las existencias,

acorralada en las conductas, perseguida en los sueños; se la sospecha en las menores locuras, se la persigue hasta los primeros años de la infancia; pasa a ser la cifra de la individualidad, a la vez que permite analizarla y torna posible amaestrarla (pp. 176177).

\footnotetext{
${ }^{2}$ Por patriarcado se entiende: la organización jerárquica masculina de la sociedad y, aunque su base legal institucional aparece de manera mucho más explícita en el pasado, las relaciones básicas de poder han permanecido intactas hasta nuestros días. Las raíces del patriarcado se encuentran ya manifiestas a través de la fuerza y el control masculino en los propios roles reproductivos de las mujeres. (Eisenstein. 1977, pp. 88-89).
} 
A propósito, Sau (1995) afirma que la maternidad no es una construcción o creación de las mujeres, en la medida en que no asumen roles sociales. La autora considera que en la medida en que las mujeres no tomen decisiones de carácter social sobre todos los asuntos, en especial, sobre aquellos en los que están íntimamente involucradas, como es el caso de la maternidad, las mujeres no son seres sociales sino "rebaño humano" que no ha superado el estado de naturaleza. En consecuencia,

su conducta no es social ni ética, no tiene una intención ni una dirección social que le de sentido. No es parte contratante, así que otros contratan, dirigen y tienen intenciones por ella, reducida a la pura animalidad de sus funciones biofisiológicas. $Y$ es desde este punto de vista que nos permitimos afirmar categóricamente que la maternidad no existe (Sau, 1995, p.177-178).

Para autora (Sau, 1995), la sociedad patriarcal concreta su poder sobre las mujeres, al apoderarse de sus cuerpos y del producto de los mismos: vidas humanas y trabajo reproductivo. Como contrato masculino, la distribución del trabajo, tanto como la del tiempo libre, la planificación del mundo productivo, y la planificación de la reproducción humana, o sea, el control sobre la demografía, se vuelve un asunto que no concierne a todos y todas, o a las mujeres en la medida en que les afecta de forma específica, sino del colectivo masculino que es quien toma las decisiones al respecto.

Con respecto a la formación de las identidades femeninas y masculinas, el orden político de dominación patriarcal construye a las mujeres como seres marcadas por la incompletud, la ilimitación y la inferioridad, subordinadas y dependientes de los hombres, conducidas por ellos; aseguradas en quienes dan sentido a sus vidas y como habitantes tutoreadas en un mundo que ya tiene dueño. Por lo tanto, ser mujer en el mundo patriarcal implica vivir a contracorriente, desde una condición inferiorizada, a partir de la cual, los hechos de las mujeres son desvalorizados o invisibilizados (Lagarde, 1997, p. 55).

Molina (1994) afirma que una de las características más llamativas del patriarcado, es la forma de poder, que se concreta en la capacidad que tiene para asignar los espacios de lo femenino en lo privado, en el sentido de que se hurta a la presencia de los demás, primero porque representa el reino de la necesidad y, segundo, porque no tiene relevancia. 
Pero, si las mujeres no se sienten dueñas de su cuerpo, se presenta una dificultad añadida en la manera de vivir la maternidad. Sau (1995), afirma, si la mujer no tiene poder de decisión sobre su cuerpo, convierte a la madre en una función de padre, su cuerpo y ella misma trabaja para el patriarcado. De este modo, la relación primaria e indispensable entre la mujer y lo que crece o ha crecido en su seno no es suficiente para calificar este vínculo de maternidad en el sentido sociocultural de la palabra. Si dar la vida no es todavía un riesgo cultural que cada mujer corre voluntariamente porque así lo ha decidido, esa vida será dada de forma natural y espontánea, donde la mujer obra como medio para un fin masculino (Sau: 1995).

Vemos cómo se construye, alrededor de la maternidad, una ética de la responsabilidad. Gilligan, (1982) propone que esta ética tiene entre sus características la organización de la vida de las mujeres, en respuesta de la percepción, sobre las necesidades de los demás. En este sentido, ejercer el control en la toma de decisiones es un riesgo, siendo posible asignarles el calificativo de egoístas y, por tanto, moralmente peligrosas; siendo que la virtud está en el autosacrificio, lo cual complica el curso del desarrollo femenino, enfrentando la cuestión moral de la bondad contra las cuestiones adultas de la responsabilidad y la elección.

Pero la mujer madre se convierte en un ser ambiguo y ambivalente y la maternidad en una utopía. Aquella parte de la socialización que el padre no hace, la delega a esa figura mediadora que se conduce, en realidad, como padre en femenino. Las madres introducen a los hijos e hijas en un sistema de representaciones que, entre otras cosas, oprime a las mujeres y bloquea los valores sociales de la maternidad, hasta el punto de desplazarla para dar lugar a la figura única del padre como rector y dirigente de los destinos de mujeres y de niños (as). De esta forma, se reproducen las condiciones del contrato social masculino en el espacio íntimo de la crianza.

\section{La educación y la transmisión cultural.}

La educación (formal y no formal) como una de las instituciones de la sociedad, produce y reproduce las creencias y valores de la sociedad; por ello no es de extrañar que en el caso de la maternidad, como el deber ser que la sociedad patriarcal les asigna a las mujeres, también sea producida y reproducida en los procesos educativos. 
En el caso de la educación formal, partimos de la premisa de que la educación es parte de un proceso de transmisión cultural que contribuye a la interiorización de elementos y valores de la cultura dominante, con una función orientada al control simbólico y social. La transmisión cultural es entendida como la transmisión del capital cultural acumulado, que se lleva a cabo de generación en generación, siendo en algunos casos, reelaborado o renovado. Esta labor de inculcar o reprimir valores se presenta encubierta en el discurso de las políticas, ocultando parte de su función y naturaleza.

La noción de capital cultural contribuye a explicar el proceso de reproducción social por la vía del sistema educativo, en tanto legitima formas de conocimiento, creencias, valores, actitudes, lenguaje y estilos de vida que constituyen aspectos centrales de la cultura dominante. En consecuencia, el sistema educativo cumple una función socio-cultural e ideológica en la formación de las personas.

Facio y Fries (2002, p. 44), expresan que

el sistema patriarcal presupone que el dominio masculino es un fenómeno universal y natural, sustentado por la religión y la ciencia. Considera lo masculino como paradigma de lo humano. Crea instituciones que sustentan este dominio, como el sistema jurídico, la educación, el lenguaje, etc. las cuales mantienen y perpetúan la desigualdad entre hombres y mujeres, enfatizando la superación de los primeros.

Estas desigualdades se especifican en la formación de las niñas, educadas para que reproduzcan cualidades de servicio y subordinación atadas a una maternidad en la que prevalece el sufrimiento, un afecto infinito hacia el otro que impide quererse a sí misma y el control del erotismo femenino, bajo la exaltación de la virginidad. Entre tanto, a los niños se les forma para que dominen a las mujeres y desarrollen su virilidad participando en el mundo de lo público.

\section{Aproximación metodológica}

\subsection{Preguntas de investigación}

En nuestro marco teórico hemos desarrollado la propuesta de que la maternidad es un concepto que se ha construido socialmente, a lo largo de la historia de la cultura occidental. Esto querría decir que la maternidad, como construcción social, sigue el mismo camino de 
las representaciones sociales de mundo y de cultura que los individuos elaboran a partir de una praxis cotidiana. De manera que las acciones cotidianas de los individuos, adquieren un significado gracias a la interacción social de la cual forman parte.

Algunas preguntas de la investigación fueron las siguientes:

¿Cómo el niño y la niña comprenden la maternidad como construcción social?

¿Cómo la escuela trasmite y contribuye en darle sentido a esta construcción de la maternidad que hacen los niños y las niñas?

¿Es posible que las mujeres -madres- de grupos de educación no formal puedan construir una comprensión de la maternidad como "proyecto humano" y no como una función natural?

\subsection{Tipo de estudio.}

Nuestro estudio se enmarca en la tradición de la investigación cualitativa, con un enfoque feminista. Esta elección se debe a nuestro interés por la comprensión de los significados atribuidos por los sujetos a la maternidad. La investigación de enfoque cualitativo nos permite tomar en consideración las creencias, valores, visión del mundo, interpretaciones y significados que los y las participantes tienen en relación con el concepto de maternidad.

Nuestra investigación ha implicado un desarrollo gradual del tema y del acto de investigación, el cual es tanto reflexivo como flexible.

Nos interesó aproximarnos a lo que un grupo de niños y niñas pudiera informarnos sobre lo que para ellos es "una madre".

Nos centramos en aquello que expresaron los niños y las niñas de nueve años, sobre la maternidad como un concepto que se construye en la interacción con los otros y, desde luego, con sus propias madres y otros referentes maternos.

Algunas puntuaciones pertinentes con el proceso de investigación con niños y niñas de nueve años son las siguientes:

- los niños y las niñas son coactivos (as),

- elaboran sus propias teorías del mundo en general y sobre el funcionamiento social en particular, 
- pueden construir a partir de su experiencia pero también pueden generar ideas totalmente nuevas que les son propias,

- los niños y las niñas expresan sus sentimientos y pensamientos a través de lo que hacen y de lo que dicen, es decir, por medio del lenguaje corporal, de la expresión artística o diciendo las cosas en forma oral o escrita. A partir de estímulos concretos, dibujos o fotografías pero también a partir de narraciones y representaciones. (Kuhn, 2003)

Estas puntuaciones nos hacen pensar que la técnica del dibujo temático y enfocado junto con la entrevista focalizada, constituye la mejor forma de indagación con niños y niñas.

\subsection{Técnicas de recolección de la información}

\subsubsection{El dibujo temático}

Nos interesan las narraciones que los niños y las niñas puedan elaborar al respecto de la maternidad, partiendo de dibujos que la evoquen, lo que para ellos y ellas significa ser madre. Esta intervención metodológica es posible, si consideramos que la sociedad como sistema social se le presenta a los niños y niñas estructurada simbólicamente. Es decir, cuando los niños y niñas hablan del funcionamiento social, lo hacen refiriéndose a una especie de texto construido en la interacción, lo cual les permite desarrollar su propia lectura del mundo. Su participación es co-activa con su entorno social, el niño y la niña desarrollan sus propias versiones del mundo, al tiempo que lo comprenden y lo interpretan.

La consigna inicial para realizar los dibujos fue: "Una madre es". Posteriormente los niños y niñas dibujaron "Un padre es" lo que nos permite realizar algunas comparaciones posteriores entre las descripciones que los menores aplican a la maternidad en relación con la paternidad.

\subsubsection{La entrevista focalizada con niños y niñas o entrevista simbólica}

En cuanto a la entrevista focalizada como un tipo de entrevista episódica cualitativa, nos proporciona

la oportunidad de aprehender específicamente el punto de vista de los niños y niñas sobre sus vidas, sus deseos, sus intereses, procesos de aprendizaje, problemas y temores con la familia y las relaciones con el grupo de pares y del medio ambiente en el que viven (Kuhn 2003, trad. libre por las autoras). 
El punto central que aquí interesa resaltar, es que esta técnica de entrevista nos permite volver a preguntar para que los niños cuenten sobre situaciones en intervalos regulares. En la entrevista, cualquier secuencia pregunta-respuesta es combinada, con un "volver a preguntar" para que cuenten de nuevo.

Cuando combinamos "contar y dibujar" sobre un tema, nos introducimos en lo que Heinzel (cit. por Kuhn, 2003) Ilama forma de "entrevista simbólica".

Al elegir un dibujo como un modo para acercarnos a la realidad de los niños y niñas, lo hacemos debido a que la literatura informa que éstos cuentan con un impulso natural para reproducir, es decir, no necesitan estímulos especiales para dibujar, tan solo dibujan.

Estas manifestaciones espontáneas pueden ser iniciadas por instrucciones para así llegar al pensamiento y sentimientos de los niños y de las niñas, de manera que el dibujo se hace comunicación en acto.

El instrumento de Tschanz y Krause llamado "Dibujo temático de niños" creado en 1992 (cit. por Kuhn, 2003) sirve para investigar ideas de los niños y niñas acerca de estados presentes, futuros y condiciones del mundo en donde viven, en otros términos, es un medio para manifestar la realidad vivida por éstos. Kuhn (2003) cita algunas investigaciones que han tratado sobre las ideas de los niños (as) acerca de sus familias a partir de dibujos (son ejemplos de estas investigaciones; Bremgräser, 1970; Dussa 1980, Kaufmamm 1988), de esta forma se ha estudiado las concepciones sobre el mundo del trabajo, sobre sus deseos o sobre problemas existenciales del presente y del futuro o sobre el ideal que los y las menores tienen sobre el o la docente.

Nuestra decisión metodológica se centró en el tópico "Una madre es...” y consideramos que a partir de la originalidad de los niños y de las niñas y de su individualidad, se podría crear un espacio creativo para desarrollar el tema.

Elegimos esta forma de entrevista por tres ventajas:

Los niños y las niñas se centran en el tema de interés y pueden expresar su punto de vista sin la influencia de las entrevistadoras, sin estar presionados por el tiempo y sin la tensión para hablar. 
El tiempo entre el dibujo y la entrevista provee a las entrevistadoras la oportunidad para elegir aquellos quienes por sus dibujos, se estima, tendrán ricas conversaciones.

Es posible trabajar con una mayor población de niños (as) que solamente dibujan pues no serán entrevistados (as).

\subsubsection{Grupo Focal}

Se utilizó esta técnica con las mujeres integrantes de un grupo de educación no formal del Programa "Creciendo Juntas" bajo la coordinación del Instituto Nacional de Aprendizaje (INA).

Se elaboró un instrumento de entrevista para facilitar la generación de ideas, con dos objetivos, uno como instrumento autoadministrado y, el segundo, como instrumento de reflexión en subgrupo.

Esta actividad fue grabada en audio y video.

\subsubsection{Entrevista dirigida para la docente y la facilitadora}

Se construyó un instrumento de entrevista para la docente del grupo de niños y niñas así como para la facilitadora.

\subsection{Población}

Nuestra población la constituye un grupo de 26 estudiantes de tercer grado de primaria (15 hombres y 11 mujeres) de la educación general básica (educación formal) en una escuela urbano marginal del Área Metropolitana. Esta población participó elaborando solamente los dibujos, y de ellos se seleccionaron 5 niños y 5 niñas, para realizar la entrevista.

Un grupo de 17 mujeres participantes en el programa "Creciendo Juntas" del IMAS en educación no formal del Área Metropolitana, quienes durante 4 meses (3 de marzo al 15 de julio) recibían una capacitación, una mañana por semana, por parte del INA. Con el objetivo de prepararse en una formulación de proyecto de autosostenimiento. Todas estas mujeres son madres que tienen varios hijos (as). Son de bajos recursos, y algunas tienen pareja.

También fue entrevistada la docente del aula del tercer grado y la facilitadora del grupo del programa "Creciendo Juntas". 


\subsection{Procedimiento de contacto con nuestra población del estudio.}

\subsubsection{Trabajo de campo con los niños y las niñas}

Luego de realizar el contacto con la Institución Educativa elegida ${ }^{3}$, se estableció un día para que un grupo de niños y niñas de tercer grado elaboraran dibujos bajo el tema: "Una madre es..." y "Un padre es..."

La entrevista se dio una semana después de la elaboración de los dibujos, bajo la modalidad de entrevista simbólica. Los niños y niñas debían contar con nueve años cumplidos, edad correspondiente al tercer grado.

Debido al tiempo de trabajo con el que se contó en la escuela, se entrevistaron a los niños y las niñas de dos en dos, procurando generar narraciones a partir del dibujo de cada uno de los menores. Para la entrevista se contó con una guía de preguntas para verificar que los temas que nos interesaban fueran tratados por los niños y niñas.

\subsubsection{Entrevista dirigida con la docente}

La entrevista con la docente, se realizó durante la misma semana de entrevista a los menores (diciembre 2003). Estas tuvieron lugar en la escuela, y para ello, se contó con una guía de entrevista. La misma fue grabada.

\subsubsection{Grupo Focal con mujeres del sector de educación no formal.}

Desde el marzo del 2004 se iniciaron las gestiones para concretar la realización del grupo focal con las mujeres, el 26 de mayo de ese mismo año, luego de un primer contacto con el grupo, en el cual se les presentaron los objetivos de la investigación y en qué consistiría la actividad.

\subsubsection{Entrevista dirigida con la facilitadora}

Esta entrevista se realizó una semana después del trabajo con el grupo de mujeres (junio 2004). El instrumento utilizado fue el mismo que se usó en la entrevista con la docente. Esta tuvo lugar en el mismo sitio donde se reúne con el grupo de mujeres. 


\subsection{Transcripción y sistematización}

Las entrevistas a los niños y a las niñas, a la docente y la facilitadora, fueron transcritas. También fue transcrita la información obtenida en el desarrollo del grupo focal, el instrumento individual y grupal y el video. Posteriormente, se sistematizó la información obtenida durante la entrevista con los niños y niñas, así como la información obtenida a partir del trabajo con el grupo focal.

\subsection{Análisis de los resultados}

Para realizar el análisis de la información de nuestra población, se establecieron cinco dimensiones de trabajo:

La dimensión descriptiva. Es un nivel básico que hace referencia a adjetivos calificativos acerca de lo que "Una madre es..."

La dimensión moral-ética. Aquí consideramos las apreciaciones que se refieren a la calificación de lo que una madre es, a partir de la valoración que los niños y niñas hacen de ella en términos de bueno y malo.

La dimensión sobre la función de la madre. Hace alusión a la comprensión que los niños y las niñas han elaborado al respecto de la organización familiar, según las funciones que realizan los miembros de este grupo.

La dimensión del cuidado. La maternidad ha sido definida por los niños y niñas, e incluso por la docente y por las mujeres entrevistadas, fundamentalmente por la dimensión afectiva que le da sentido a las funciones que realiza la madre. En este sentido, la dimensión nos aporta, la construcción semántica acerca del cuidado materno.

La dimensión sobre la función de la escuela. Recuperamos en esta dimensión lo que la Escuela como Institución Social reproduce sobre la maternidad como creación patriarcal. Esto es, en términos de asignación de roles y discursos acerca de lo que una madre, una niña y un niño deben ser.

3 Los criterios de selección de esta escuela fueron, pertenecer al Área Metropolitana, lo cual garantizaba el acceso para las investigadoras. Otro criterio fue el pertenecer al sector educativo 


\section{Discusión teórica a partir del corpus de entrevistas}

Por razones de espacio no es posible presentar cada uno de los análisis realizados a las entrevistas que se desarrollaron en esta investigación. Sin embargo, el lector que quiera ampliar acerca de este análisis, puede consultar el informe final de investigación que se encuentra en el Instituto de Investigaciones en Educación (INIE).Aquí presentamos de manera sintética el análisis de las entrevistas.

\section{La idealización}

El marco de referencia de nuestra propuesta teórica, sostiene que la maternidad es un concepto que se ha construido socialmente, a lo largo de la historia de la cultura occidental. Esto querría decir que la maternidad como construcción social sigue el mismo camino de las representaciones sociales de mundo y de cultura que los individuos elaboran, a partir de una praxis cotidiana, de una experiencia concreta que ayuda a organizar, justificar el funcionamiento social como forma de ver el mundo y a dirigir las acciones del individuo en la sociedad en donde vive. De manera que como imaginario social e individual, les da sentido a las prácticas sociales y privadas de las mujeres. Sus acciones cotidianas adquieren un significado, gracias a la interacción social de la cual forman parte.

La sociedad occidental con la herencia patriarcal, les asignó a las mujeres la función de la maternidad como práctica privada y, por el mismo movimiento, la excluyó de los procesos de generación cultural. Sin embargo, la maternidad representa un lugar de contradicción, pues mientras la mujer es excluida de la creación cultural en términos de su participación pública en la sociedad, la maternidad también atrapa a las mujeres entre "tules y celofanes".

En esta construcción de la maternidad, la mujer es definida desde fuera de sí, por la asociación mujer=madre, sometiéndola a una especie de alejamiento de sí misma, lo cual permite construir un sobreentendido al respecto del proyecto de vida femenino, Ser Madre. Se define así una identidad para la mujer en términos de su maternidad, como la madre que es, la que pudo ser, la que será.

Desde edades tempranas las mujeres empiezan a construir una experiencia de maternidad, asumiendo tareas de adultas, las cuales están asignadas tradicionalmente a la mujer, como lo son los oficios domésticos y el cuidado de los otros; por ejemplo, los hermanos menores - 
como lo testimonian algunas de las niñas durante las entrevistas-, es así como la mujer va siendo asimilada a la maternidad y ésta a las tareas domésticas y de cuidado, y así se define a partir de ellas.

El cuidado, el servicio, la dependencia, el amor, y el Eterno femenino, son las características que niños, niñas y mujeres utilizan para describir la función de la mujer-madre. A la pregunta "Una madre es..." los niños y niñas respondieron,

Una madre buena da cariño, amor y siempre contamos con ella y está para ayudarnos S (niño)

Yo siempre... tuviera que estar a la par de ella para que ella me haga cariño y de salud $\mathrm{K}$ (niño)

No (le dice) malas palabras a los bebés y no pegarles a los bebés recién nacidos. A (niña).

Por otra parte, para la mayor parte de las mujeres entrevistadas (13/17), el ser madre no se construye sino que es un instinto. Sin embargo, algunas (4) reconocen que la sociedad obliga a las mujeres a ser madres por la violencia, la pobreza y el analfabetismo. A pesar de ello, dos de estas últimas consideran que la principal función de la mujer, es ser madre.

A lo largo del análisis de las entrevistas, identificamos que la construcción del concepto de maternidad, se produce en el contexto de una encrucijada de fuerzas: las psicológicas, socio-culturales e ideológicas. La maternidad se vive con sentimientos ambiguos, especialmente por la condición de vulnerabilidad social de las mujeres entrevistadas, ya que las mujeres continúan reproduciendo en su discurso, los mitos al respecto de ser madre; pero con la vivencia acerca de lo difícil que les resulta serlo.

Los testimonios de las mujeres, ilustran la contradicción entre vivencia e idealización de la maternidad. Por un lado, vivida como regalo de Dios, pero por otro, desprovistas del reconocimiento social acerca de lo que el ejercicio de la maternidad aporta a la organización de las sociedades. Una de las mujeres entrevistadas afirma:

la maternidad es una carga muy dura, algo muy grande, muy lindo y los hijos lo motivan a uno, los hijos son el mayor triunfo en la vida, por ellos me levanto y me 
enfrento a la realidad y le pido a Dios vivir un día más cada día para que ellos lleguen a ser adultos de bien a la sociedad en la que vivimos.

Para estas mujeres la sociedad aumenta las demandas sobre las madres, pues espera lo mejor de ellas, (6/17), pero al mismo tiempo, afirman (14/17) que la sociedad les "recrimina" sin conocer la realidad que éstas viven para seguir adelante por los hijos.

El mito que se construyó alrededor de la abnegación de la madre, permite su idealización, al tiempo que una especie de negación al respecto de su trabajo como actividad productiva, pues las mujeres también producen riqueza económica y social, a partir de su trabajo reproductivo.

No obstante, cuando en nuestra investigación se planteó la propuesta de una remuneración por el trabajo que realizan las madres, tanto en mujeres como en niños y niñas, provocó tal disonancia que para algunas (os) es difícil aceptarlo. Como lo observamos en las entrevistas con las y los participantes en este estudio, ya que si la maternidad es bendición de Dios, función de la mujer y en su instinto está el ser madre, la maternidad lleva en sí misma la retribución como "regalo de Dios".

Proponer este tipo de pago provoca desconcierto, pero además parece provocar culpa, ya que como observamos en el análisis, pagar por el ejercicio de la maternidad es contra natura. En este sentido, la respuesta de las madres aunque, en un nivel de razonamiento distinto de los niños y de las niñas, llega a la misma conclusión: la maternidad en sí misma es una retribución para la mujer.

Lo dicho por la docente es aquí ilustrativo, ella afirma: yo nunca pensé en que me tenían que pagar por cuidar a mis hijos (...).

Pero también encontramos que para muchas mujeres es la carencia económica la que las hace pensar en un pago por la maternidad, el cual se considera una ayuda para los hijos (as) y no un reconocimiento por su desempeño. Por ejemplo, algunas madres (8/17) entrevistadas sí estarían de acuerdo con una retribución salarial para que madre e hijo surjan o para ver a los hijos, pues la situación de pobreza de estas mujeres es extrema. 
Por otra parte, esta carencia económica las hace considerar su inserción en la vida social productiva y no sólo reproductiva, construyendo un proyecto de vida asociado con el estudio, pero de nuevo, en función de sus hijos (as) y no de sí mismas. Por ellas mismas no llegan a visualizarse como sujeto de deseo. Por ejemplo, algunas mujeres (7/17) hubieran querido estudiar o hacer otra cosa en la vida, en lugar de ser madres, aunque a otras (9/17) les gustaría ser madres y haberse preparado con estudios.

\section{¿Cómo se concibe la maternidad?}

En las entrevistas realizamos a las niñas y a los niños, observamos que desde esta edad, se han organizado en ellos, los referentes de la asociación mujer=madre. En las niñas, por ejemplo, la madre es para el cuidado de los demás y como niñas, ellas ya están reproduciendo e incorporando los imaginarios que las designan como mujeres -cuidando hermanos y padres- pero no cuidando de sí mismas.

...(la madre) es la que tiene que estar en la casa" (léase, hacer oficio) "La mamá (hace los oficios) porque la mamá es la que tiene que estar en casa. A. (niña)

...mi mamá hace los oficios. J. (niño)

Mi mamá, yo le digo así a la muchacha que me cuida. K. (niño)

Además, la maternidad y el oficio doméstico se encuentran tan ligados que, cuando alguien se encarga de ellos, como "la muchacha que cuida", se merece el calificativo de madre.

Pero la maternidad también se encuentra asociada con lo femenino, por lo que la responsabilidad por los oficios domésticos también se trasmite a otras mujeres, que se supone, llegarán a ser madres:

...Ella (mamií) nos enseñó a tener una vida, nos enseñó su amor y a cocinar...

Yo cocino cuando mi mamá está enferma. A veces yo le hago un té de manzanilla y aplancho mi uniforme y la ropa de mi papá. E. (niña) 
El hombre (padres o hermanos) solo asumen estas labores por defecto, es decir, cuando la madre no está disponible:

mi mamá cuando hace algunas vueltas mi papá limpia. D. (niña)

Yo (lavo y plancho la ropa) de toda la familia, menos la de mi hermano, él se levanta como a las 5 a planchar (mi hermano trabaja). D. (niña)

El cuidado de los hijos (as), específicamente cuando están enfermos, es una labor que de nuevo es preferible que realice una madre o una mujer. Solo cuando se sale del ámbito doméstico la labor la asume el padre u otro hombre, así se construyen lugares simbólicos para las mujeres y para los varones,

Mi mamá y mi papá (me cuidan cuando estoy enfermo). Cuando mi papá no está, mi mamá lo llama al trabajo y él va a cuidarme al hospital. K. (niño)

Sin embargo, la valoración social y personal para la mujer que sostiene este mito de abnegación tiene un precio: cuidar no sólo a sus hijos (as) sino también al marido, a los hermanos, a los alumnos y a todo lo demás que se encuentre en su campo de servicio doméstico. A propósito nos aporta la entrevistada quien trabaja como facilitadora en el grupo de mujeres:

(...) A nivel del programa lo tenemos claro, recibimos un taller de la relación madre-hija, el que me ayudó a aprender que nosotras no podemos cargar con las necesidades emocionales de esas mujeres como si fueran nuestras hijas. A veces nos hacemos bromas (entre facilitadoras) una compañera me dice que son mis hijas, cuando llaman mucho y yo les digo que no son mis hijas. No sé si las otras (facilitadoras) lo tengan claro, el equipo (con el que ella trabaja) sí. Sí se vió cómo las mujeres hemos sido socializadas que nos sentimos la mamá del pollo, la mamá del perro, la mamá de la pulga, la mamá de todo el mundo o cómo se revierte la relación madre-hija a que la hija asuma la maternidad de la mamá. Tenemos claro cuál es la función de este proceso de capacitación, no caer en situaciones que lleven a eso, es importante promover en ellas la iniciativa, el desarrollo, la independencia. Es importante que ellas den lo que tienen. 
Solo por medio de un giro auto reflexivo, la mujer se percata del costo personal de asumir la maternidad como abnegación y finalidad.

Para las mujeres su feminidad es reducida a la sexualidad y, esta a su vez, a la maternidad. Esta última ha sido tanto el acceso al goce como la negación del mismo. Si la feminidad y la sexualidad se desligan del acto de engendrar, se abriría la posibilidad de la realización personal en un acto de generación creativa cultural, siendo la maternidad igualmente un acto creativo.

Con ello proponemos la deconstrucción de la maternidad como concepto y como vivencia de las mujeres. Pues la experiencia de la maternidad dentro del Ideal, obtura la posibilidad de concebirse mujer sin ser exclusivamente madre. Este acto de deconstrucción implicaría definir lo femenino sin el condicionamiento de la maternidad y una maternidad sin el condicionamiento del Ideal.

\section{La maternidad en la escuela}

La escuela como institución social reproduce la maternidad como creación patriarcal. En su acción pedagógica, la escuela participa de la formación social o cultural de los niños y niñas. La transmisión de valores y pautas de comportamiento se refuerzan en este escenario escolar, donde se reproducen en pequeña escala el macrosistema y sus relaciones.

En los docentes, por el ejercicio de su "autoridad pedagógica", es en quienes se apoya o vehicula el discurso cultural arbitrario acerca del funcionamiento del mundo. También sabemos la importancia que reviste en la formación de niños y niñas, pues debido al fuerte lazo afectivo que se establece entre ellos, su credibilidad como representante de la sociedad es mayor.

Pero además observamos que la organización de la escuela reproduce roles que podrían recordar la vivencia de algunos niños y niñas en sus familias, siendo la educación una profesión que la ejercen fundamentalmente mujeres; son los varones quienes se encuentran en puestos de mando (directores). Bajo la dirección masculina, algunas escuelas funcionan como representantes de la organización doméstica familiar. Las leyes de la escuela son del gobierno masculino; el cuidado y la crianza un asunto de mujeres. 
La escuela alberga en su mayoría a mujeres trabajadoras que se relacionan con sus estudiantes, según el discurso tradicional de la maternidad, de lo privado y de lo doméstico. Ellas transmiten conocimiento, pero también protegen y dan consejos; los directores en cambio, ponen los castigos más fuertes y, en las ocasiones en las que la docente no puede hacerse cargo de situaciones disciplinarias se envían a los niños y niñas (principalmente niños) a la Dirección -así lo atestiguan los niños y las niñas entrevistadas-.

En la escuela, la maestra también se asemeja a la madre. Esta relación si bien no es explícita en las entrevistas con las niñas y los niños, sí se puede observar en la entrevista personal con la docente quien declara:

...el papel de la mamá es guiarlos (a los hijos) al igual que la maestra, ese es el papel de nosotras.

La escuela es un lugar para la mujer donde el ejercicio profesional se traslapa fácilmente con el ejercicio de la maternidad. La docente es así una figura esencialmente para el cuidado, realizando en esta función un nuevo "maternaje". Ella afirma, acerca del rol de la docente en relación con el de la madre:

... claro que tienen mucha relación porque el papel de maestra es de una persona guía, que los va guiando a ellos al igual que me imagino que la mamá, el papel de la mamá es guiarlos al igual que la maestra, ese es el papel de nosotras. (Ejemplifica) (...) mucho chiquito me dice mami en lugar de niña, verdad, se le safa: "Ay se me olvidó, pensé que estaba con mami", me dicen. Los chiquillos lo ven a uno como mamá, lo relacionan con la mamá dependiendo de la afinidad que tengan aquí con los profesores. Me imagino que si tienen a un hombre como profesor, como maestro, van a decir, van a relacionar más al papá con el profesor. En cambio a la maestra, van a relacionar más a la mujer con la mamá.

El "maternaje" que ejercen las mujeres donde quiera que se encuentren -vida pública o privada- asegura el cuidado hacia otros, pero con ello la dependencia de quienes son cuidados. A diferencia del rol que asume la facilitadora en los procesos de formación no formal. Ella comenta:

en la educación formal la maestra asume bastante el rol de madre, sobre todo en la primaria. La maestra lo asume, va condicionada a ser lo dulce y cariñosa que los niños necesitan y aún más buscan cubrir esos espacios cuando las situaciones familiares son difíciles. De parte de 
los alumnos siendo que hay una tendencia de los seres humanos a buscar mamás y de las mujeres a comportarse como mamás. Tal vez lo hice en algún momento, pero ahora creo que el ser humano tiene la capacidad suficiente de dar y buscar lo que requiere. Esa maternidad es producto de la socialización. Se le ha dado a la madre el papel de: insustituible, irremplazable, como lo mejor, el ser más amado; pero esto en términos de cuidados, de dependencia, no de amor y afecto, porque el amor y el afecto están ligados a los actos (...)

Sin embargo, para la facilitadora no existe relación entre las funciones de la madre con las suyas; más bien, llama la atención acerca de la importancia de mantener distancia entre ambas:

(...) hay que tener mucho cuidado en no caer en maternalismos porque las mamás se dedican a proteger. No sé si -las señoras del curso- han pensado en que soy como una hija, por la edad. Hay que aclarar que el curso es un proceso constructivo donde se puede desarrollar amistad, pero no dependencia. Se habla de enriquecimiento personal, empoderamiento y en un rol de maternidad no se da eso, se da dependencia y centralización del poder. Yo no tengo responsabilidades, obligaciones afectivas como las que se imponen en una relación de maternidad. No hay que confundir y tratar de que de la mezcla con los sentimientos de las personas.

No obstante, rescatamos el esfuerzo de la docente para transformar ciertos patrones de género que experimentó en su infancia y aún en su vida adulta y que no desea para sus estudiantes. Lo que la induce a proponer cambios en los procesos educativos. Ejemplo de estas vivencias son:

Cuando yo voy manejando, a mí me da rabia porque, cualquier cosa que pasa, "ah tenía que ser una mujer" y entonces me pitan y me pitan. Pero yo lo que tengo muy metido, pero así desde chiquitita, es que en mi casa existió mucho eso (sobre el machismo). Entonces mi mamá nos ponía por ejemplo, usted vaya y le aplancha el pantalón a su hermano porque él tiene que salir. Toda la tarde ahí aplanchándole la ropa a mi hermano y mi hermano en el sillón o esperando "la ropita" o mi papá también llegaba y se sentaba en la mesa y "dónde está", cuando se le olvidaba la servilleta a mi mamá o cosas así, entonces ya, no se paraba, él no se paraba a buscar la servilleta hasta que mi mamá llegaba y se la ponía. Me chocaba tanto tanto, que ahora en cualquier momento yo trato de cortar con eso. 
Todo ello la hace asumir un discurso con los niños y las niñas, donde al menos trata de hacerles ver que las diferencias tradicionalmente asignadas a hombres y a mujeres, se refieren a una construcción social y no ligado a la biología. Estos pasos son importantes en el camino necesario de la deconstrucción de conceptos mitificados socialmente como la maternidad.

La docente comenta:

Aquí el mejor promedio de la clase es un hombre, entonces los felicitamos cada vez que entregamos notas, yo los felicito aquí delante de la clase y una chiquilla dijo una vez, "ah, es por que por ser hombre es más inteligente". Entonces yo les hice la aclaración, "no, no, es por ser hombre, es porque él estudia. Ustedes tienen la posibilidad de ser igual o más que Osar y eso no quiere decir que las mujeres y los hombres seamos diferentes, o que los hombres sean más inteligentes que nosotras o que las mujeres seamos menos inteligentes que los hombres, eso depende de cada cual y entonces así yo les hago la aclaración. Pero así como 2 o 3 veces me han salido con cosas así.

\section{Conclusiones}

Nuestra propuesta ofrece una transgresión para la sociedad patriarcal como paradigma de la organización social. El repensar la definición de la feminidad conlleva, por tanto, una transformación en varios sentidos, en lo que respecta de la definición de la masculinidad y en la concepción de las instituciones sociales. La asignación de nuevos roles, tanto como el ejercicio de ellos, propiciaría la construcción de un discurso, respecto de la maternidad y de la paternidad, no en función de la biología, sino en función del compromiso con los hijos, las hijas y con el otro y la otra de la relación. De modo que la tarea de cuidar a los hijos y a las hijas, no sólo sería una responsabilidad, exclusiva, de la mujer.

Tratamos con una sociedad que, en la asignación de la función doméstica para la mujer, no permite desarrollar procesos de crecimiento personal y social. Por tanto, una deconstrucción del concepto de maternidad plantea, a su vez, la deconstrucción del concepto paternidad, quizás una utopía para consolidar una ética humana.

Pero con ello, proponemos una nueva forma de organización social que plantea retos a la estructura de funcionamiento de las instituciones, en especial, aquellas que se dedican a la educación. La educación, como carrera principalmente de mujeres, tiene el reto de transformar 
la representación como servicio abnegado, extensión de la maternidad al de la profesionalización

En una propuesta de deconstrucción es importante considerar dos ejes de trabajo:

\section{Lo afectivo-relacional (emocional) asociado con la maternidad.}

Bajo esta consideración, pensamos incluir el tema de las relaciones madre- hija. Pues la deconstrucción del discurso empieza por aclarar las relaciones afectivas que lo fundamentan. Así afirmamos que la construcción de la maternidad se realiza desde el nacimiento, a partir de la socialización primera con los cuidadores. La madre se hace con el ejemplo de la propia madre, en el cuidado de los hermanitos y haciendo labores de casa desde que son pequeñas. (12/17 mujeres opinan esto) para luego concluir el proceso al cuidar a los propios hijos (as)(10/17 mujeres también lo afirman).

2. Lo representacional-cognitivo que las mujeres construyen al respecto de la maternidad. Una pregunta importante para las investigadoras, es si es posible construir un concepto de maternidad sin restringirla a las tareas domésticas, al cuidado, partiendo de que el padre tendría que cuidar de igual forma a los hijos e hijas -que por demás, lo expresan las mujeres de este estudio-. De modo que sin tareas domésticas y sin restricción al cuidado: ¿De qué otra manera es posible construir el concepto de maternidad para las mujeres?

La mujer cuyo rol es la de facilitar procesos de formación para adultos nos muestra un paso para la deconstrucción del concepto maternidad. La referencia de esta mujer es hacia los actos de amor.

Cómo hacer de ellos una fuente nueva de definición para la maternidad, sin caer en los "peligros" de la idealización del "Amor Materno"; es un reto para las mujeres que tratan de construir una vivencia alternativa para la maternidad. Por ello, la maternidad tanto como la paternidad tendrían que procurar la independencia de los hijos e hijas, con la misma oportunidad para ambos de buscar su desarrollo y realización personal en los actos de generación cultural. 
Pero queremos hacer énfasis en el hecho de que tampoco se trata de hacer del tema de la maternidad un contenido del currículo educativo como la docente de primaria sugiere, ella nos dice:

En realidad el currículo no nos da tiempo de hablar sobre los temas, que no sea las cuatro materias básicas. A veces no nos alcanza el tiempo y a veces me resulta sumamente difícil sacar un ratito de tiempo para hablar sobre otras cosas. En realidad ellos no me han preguntado, no han mostrado interés por algunos otros temas, sobre sexo, sobre maternidad, sobre paternidad.

Nuestra propuesta, en cambio, tiene que ver más bien con una práctica que se concreta en la relación con los niños y las niñas. Ya que consideramos que la maternidad es fundamentalmente una experiencia de relación.

Y en el caso particular de la escuela, lo fundamental en la formación de los niños y niñas, está en relación directa con el potenciar un desarrollo ético y afectivo autónomo, que permita el disfrute de ser mujer y varón, en las mismas condiciones de responsabilidad colectiva.

\section{REFERENCIAS}

Alvarado, K. (1996). Una aproximación psicoanalítica a la experiencia de ser madre. Tesis para optar por el grado de licenciatura en Psicología. Universidad de Costa Rica. San José, Costa Rica

Bordieu, P. y Passeron, J.C. (1977). La reproducción. Barcelona: España: Editorial Laia.

Caamaño, C. y Rangel, A. (2002). Maternidad, feminidad y muerte. La miradas de los otros frente a la mujer acusada de infanticidio. San José, Costa Rica: Editorial Universidad de Costa Rica.

Calvo, Y. (1993). La mujer, víctima y cómplice. San José, Costa Rica: Editorial Costa Rica.

Camacho, R. (2001). La maternidad, como institución del patriarcado: representaciones y manifestaciones en obreras del sector textil. Tesis de maestría en Estudios de la Mujer. Universidad de Costa Rica. San José, Costa Rica.

Corrales, F., et. at. (1982). El papel de la mujer y la división social por sexo. Tesis para optar por el grado de licenciatura en Psicología. Universidad de Costa Rica. San José. 
De Simone, A. y Saavedra, P. (1992). Lactancia y experiencia de maternidad. Tesis para optar por el grado de licenciatura en Psicología. Universidad de Costa Rica. San José, Costa Rica.

Duarte, I. (1989). Población y Condición de la mujer en República Dominicana. República Dominicana: Centro de Estudios de Población y Desarrollo.

Everingham, C. (1997). Maternidad: autonomía y dependencia. Un estudio desde la psicología. Madrid, España: Narcea.

Facio, A. y Fries, L. (2002) Género y derecho. Colección Contraseña, Estudios de género. Antología: La construcción social de la cultura de la violencia. San José: UNED.

Fernández, A. (1993) La mujer de la ilusión. Pactos y contratos entre hombres y mujeres. Buenos Aires, Argentina: Piados.

Fonow, M. (1991). Cook. Beyond. Methodology. Feminist Scholarship as Lived Research. Indiana, Estados Unidos: Indiana University Press.

Foucault, M. (1998). Historia de la sexualidad I. La voluntad de saber. Madrid, España: Siglo XXI.

INAMU (2001). Programa Creciendo Juntas. Componente fortalecimiento personal y colectivo de las mujeres: módulo apropiándonos de nuestros saberes y poderes. San José, Costa Rica: Editorial ICER.

Kuhn, P. (2003). Thematic Drawing and Focused, Episodic Interview upon the DrawingA Method in Order to Approach to the Children's Point of view on Movement, Play, Sports at School. (Informe de Investigación de Forum Qualitative Sozialforschung/Forum: Qualitative Social Research No. 1, vol 4). Recuperado en agosto del 2003, de http://www.qualitative-research.net/fqs-texte/1-03/1-03kuhn-e.htm

Lagarde, M. (1997) Género y feminismo. Desarrollo humano y democracia. Cuadernos inacabados 25. España: Horas y HORAS

Lagarde, M. (1992). Identidad de género. Curso; Nicaragua.

Molina, C. (1994). Dialéctica feminista de la llustración. Madrid, España: Anthropos.

Olivier, C. (1994). Les fils d Oreste ou la question du père. Paris, Francia: Flamarion.

Rodríguez, R. (1994) Femenino fin de siglo: La seducción de la diferencia. Barcelona, España: Editorial Anthropos.

Rojas, Y. (1992). Transformaciones recientes en la sociedad costarricense. El nuevo rostro de Costa Rica. Heredia: CEDAL.

Sau, V. (1994). La ética de la maternidad. Mujeres y sociedad. Nuevos enfoques teóricos y metodológicos. Servicio interdisciplinar Mujeres y Sociedad. Barcelona: UB 
Valladares, B. (1994). Revisión teórica sobre los mitos de la maternidad. Revista Ciencias Sociales 65: 67-74, setiembre. Universidad de Costa Rica.

Vásquez, A.; Martínez, I. (1996). La socialización en la escuela. Una perspectiva etnográfica. Madrid, España: Paidós.

Vegetti, S. (1990). El niño de la noche. Hacerse mujer, hacerse madre. Madrid, España: Editorial Cátedra.

Villarreal, Ana Lucía. (1999). Relaciones de poder. Mujeres en la encrucijada entre trabajo productivo y trabajo reproductivo. Tesis de Maestría en Sociología, Universidad de Costa Rica, San José, Costa Rica. 